\title{
Disaggregation of low resolution NDVI data for monitoring vegetation in mining area
}

\author{
ZHANG Xuanxuan, JIA Yi, MIAO Po, ZHANG Xiaorong, MA Baodong \\ College of Resources and Civil Engineering, \\ Northeastern University, \\ Shenyang 110819, China \\ mabaodong@mail.neu.edu.cn
}

\begin{abstract}
Vegetation cover in mining area may be influenced by the exploitation and utilization of mineral resources. Because of the small size of mine goaf, coarse-resolution remote sensed images are not able to detect the variation of vegetation above the goaf. In this paper, the $250 \mathrm{~m}$ MODIS NDVIs were disaggregated into $30 \mathrm{~m}$ NDVIs by the help of land-cover map based on TM images. The result showed that the disaggregation precision is higher using the NDVI-based density slicing land-cover map than false-colorcomposite supervised-classified land-cover map. In addition, the number of selected pixels for building system of equations was discussed to reduce the amount of calculation on the premise of ensuring the precision.
\end{abstract}

Key Words-vegetation monitoring, MODIS NDVI, disaggregation, mining area, digital mine

\section{I . INTRODUCTION}

Environment and vegetation cover in mining area may be influenced by the exploitation and utilization of mineral resources[1]. For example, the resulting subsidence caused by underground coal mining technique could be very large, occurring immediately after or during mining. This significant change in the overlying ground topography can therefore cause serious problems such as damaging vegetation. It needs timely monitoring and estimation by the help of satellite remote sensing technology [2-4]. MODIS NDVI data were routinely used to monitor growth vigor of vegetation at the continental scale. However, the mining face is approximately hundreds meters long, and it needs more fine resolution NDVI Data for vegetation monitoring. To enhance the usefulness of time series of coarseresolution MODIS NDVI data in mining areas, this paper introduced how to disaggregate low-spatial-resolution images to monitor the vegetation change in mining area.

\section{II . DATA AND STUDY AREA}

The 16-day composed MODIS NDVI, with a spatial resolution of $250 \mathrm{~m}$, was set as test data. TM data acquired at the same time (August 6, 2002 ) was used to produce land-cover map and to calculate reference NDVI for validation.

This paper took Shendong mining area as an example (Fig.1). Shendong mining area locates at the junction of Inner Mongolia Autonomous Region and Shaanxi Province, Northwest China, and has annual yield of more than 100 million tons raw coal. Because of extremely poor water resources, the eco-environment is very fragile. The study area covers about 65000 MODIS pixels.

\section{METHOD}

\section{A. Disaggregation method}

Disaggregation is achieved by exploiting information about the fractional cover of each class within the low-resolution pixels, as derived from the analysis of high- resolution land-cover maps (Fig.2). Based on the linear mixing theory, it assumes that the NDVI of a mixed pixel can be calculated as the sum of mean NDVI values of the different land-cover classes within the pixel, weighted by the corresponding fractional cover[5-7]. The general equation can be written as in Eq.(1):

$$
N D V I_{i}=\sum_{j=1}^{n} f_{i j} \bullet N D V I_{j}+\varepsilon_{i}, i=1,2,3, \ldots, m
$$

where $N D V I_{i}$ is the NDVI value of the MODIS pixel $i, f_{i j}$ is the fractional cover of land-cover class $j$ in the MODIS pixel $i$, $N D V I_{j}$ is the NDVI value of land-cover class $j, n$ is the number of land-cover classes, $\varepsilon_{i}$ is residual error term and $m$ is the number of MODIS pixels. Because the number of MODIS pixels is usually greater than the number of land-cover classes, an overdetermined linear system of equations could be built for $N D V I_{j}$ and it could be solved by Ordinary Least Squares techniques. 


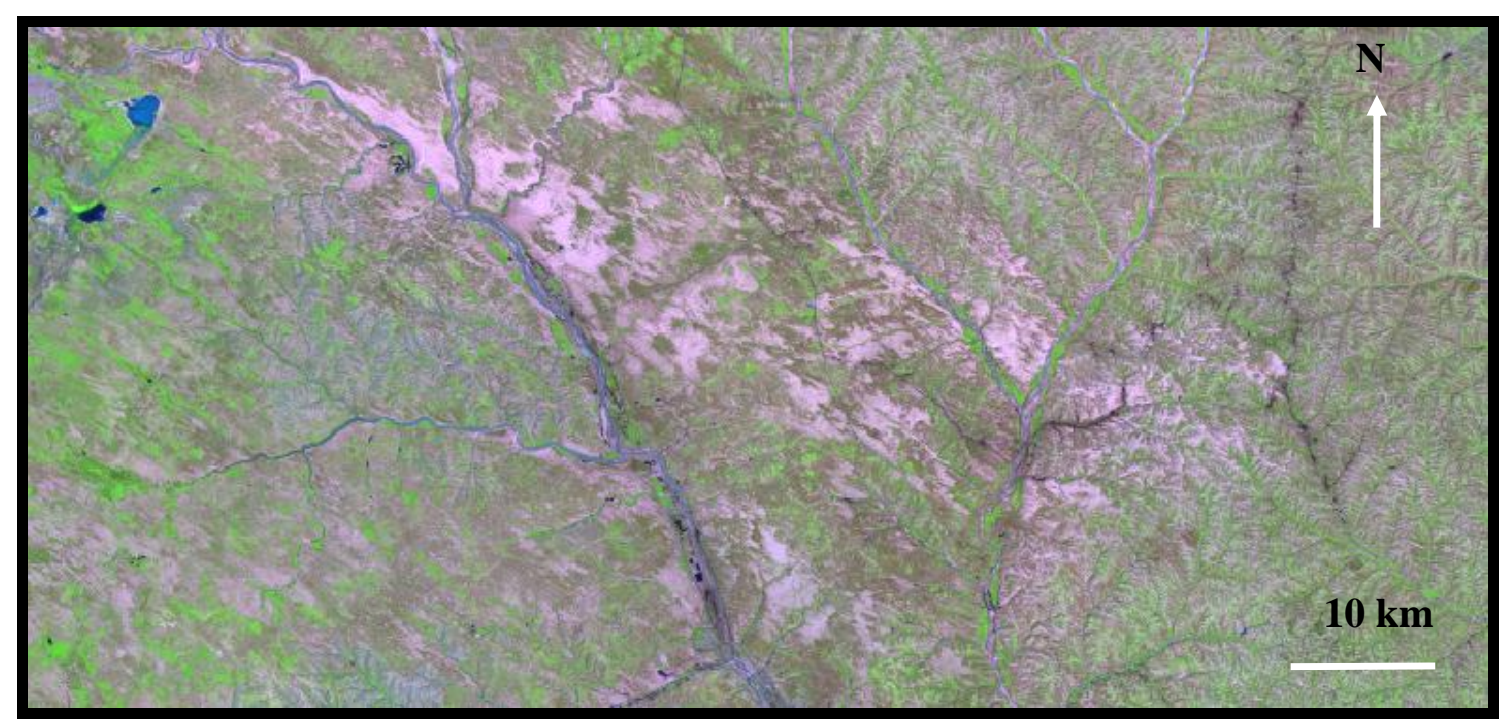

Fig.1. The study area (False-color composite of Landsat TM RGB543)

The flow chart is present below (Fig.2). Firstly, MODIS images and TM images were well co-registered, so that no further geometric correction was performed. Secondly, by using TM image, land-cover map was made respectively by supervised classification based on false-color image and density slicing based on TM NDVI image which is calculated by DN value. To get accurate result, the mining area was classified into 20 cover types. For each selected MODIS pixel, the areas occupied by the different land-cover classes are identified on the high resolution land-cover map, and TM pixels belonging to each class are extracted as fractional cover. Then it is possible to build an over-determined linear system of equations for the pixels within the whole scene. At last, with the help of MATLAB software, the system of equations was solved to generate high-resolution NDVIs.

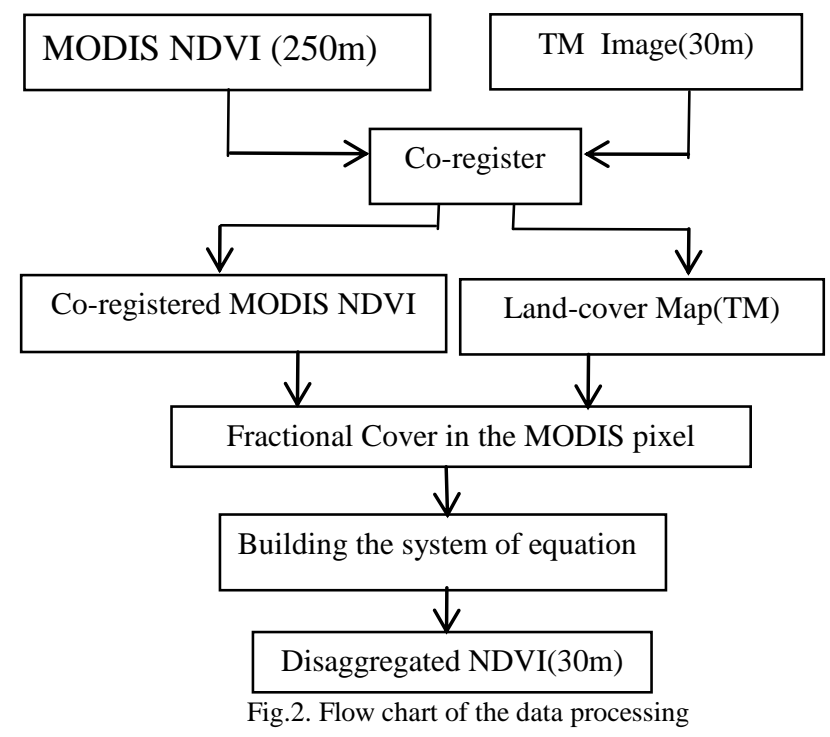

\section{B. Determining the number of selected pixels for building equations}

To solve above over-determined linear system of equations in a high speed, the number of equations should not be too large. Generally, all the pixels within the whole scene would be selected to build the system of equations. For example, the mining area image contains 65000 pixels and they can build 65000 equations. This is computationally intensive and timeconsuming. To reduce the amount of calculation, the least pixels should be selected on the premise of ensuring the precision. Step by step, different number of pixels was selected to build the system of equations. The solution of the system of equations is the NDVI value of each class. Validation was conducted by comparing disaggregated NDVI with the corresponding NDVI calculated on TM image which was first atmospherically corrected by the ENVI FLAASH module[8]. In other words, TM NDVI was set as reference data and the correlation coefficient between disaggregated NDVI image and TM NDVI image represent the accuracy of disaggregation.

\section{RESULTS}

By use of all the pixels in the image, the system of equations was solved to get the NDVI value of each class. The new disaggregated NDVI was produced (Fig.3). On the one hand, when 2000 pixels were selected for calculation, the correlation coefficient between disaggregated NDVI image and TM NDVI image is 0.753 by using supervised-classified land-cover map, while it is 0.939 by using density slicing land-cover map. It means that the density slicing method is more suitable for the disaggregation of NDVI.

On the other hand, for different number of selected pixels, the greatest correlation coefficient was 0.969 and the lowest was 
0.250(Fig.4). On the whole, the correlation coefficient increased in pace with the increasing number of selected pixels. While the number of selected pixels increased to 2000 from 50, the correlation coefficient increased rapidly. The correlation coefficient became 0.939 while 2000 pixels were selected to build the system of equations. However, the correlation coefficient did not increase obviously while the number of selected pixels was more than 2000. It meant that all the pixels of the whole scene were not necessarily selected for the calculation. Under such circumstances, 2000 pixels were enough to create relatively accurate high-resolution NDVI image.
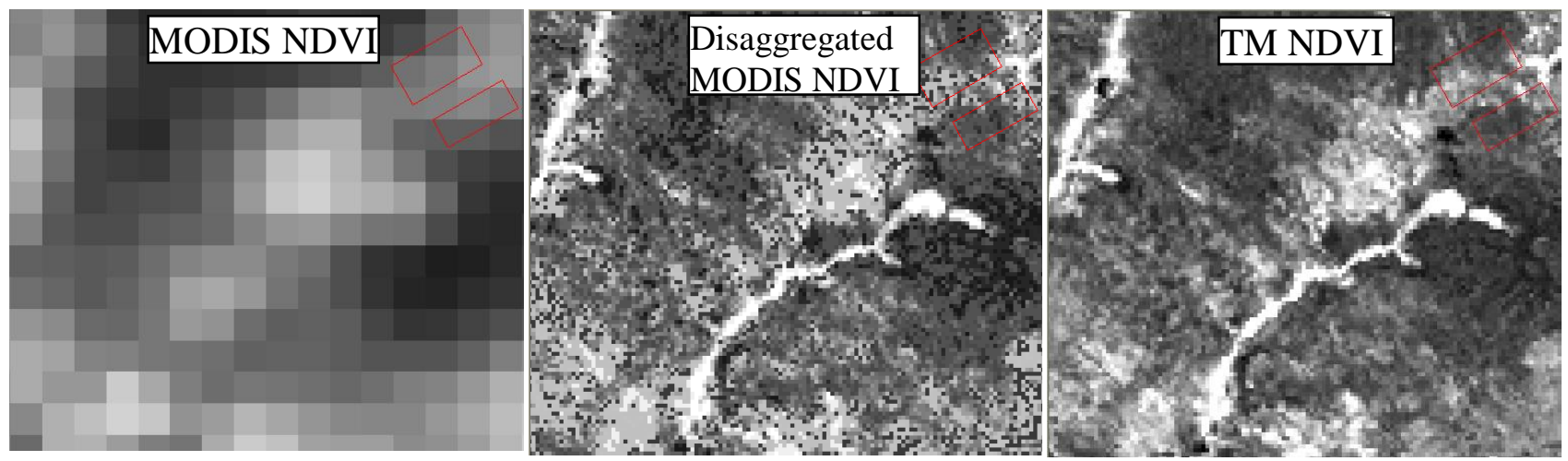

Fig.3. Disaggregated NDVI and corresponding TM NDVI image

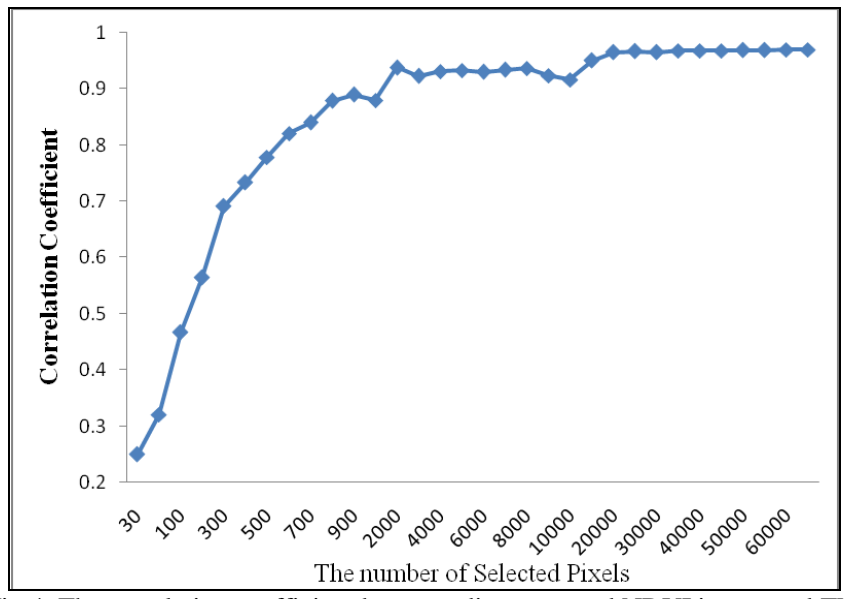

Fig.4. The correlation coefficient between disaggregated NDVI image and TM NDVI image

\section{CONCLUSIONS}

The MODIS NDVI data was disaggregated with the landcover maps derived from TM images acquired on August 6, 2002. The disaggregated NDVI was validated using TM NDVI. The result showed that the disaggregation precision is higher using density slicing land-cover map than supervised-classified land-cover map. In addition, to reduce the amount of calculation and increase the calculation speed, this paper attempted to determine the least pixels of the scene for relatively accurate disaggregated NDVI. In Shendong mining area, 2000 pixels of the whole image were enough to produce high-accuracy data. In the future, the work will focus on producing the temporally continuous high-resolution NDVI data for dynamical monitoring $[9,10]$.

\section{ACKNOWLEDGEMENT}

This work is jointly supported by the National Natural Science Foundation of China (41201359) and Training Programs of Innovation for Undergraduates (College of Resources and Civil Engineering, Northeastern University).

\section{REFERENCES}

[1] Rasim Latifovic, Kostas Fytas, Jing Chen, et al. "Assessing land cover change resulting from large surface mining development", International Journal of Applied Earth Observation and Geoinformation, pp. 29-48, 2005, 7(1)

[2] R.S. Chatterjee, "Coal fire mapping from satellite thermal IR data A case example in Jharia Coalfield, Jharkhand, India", ISPRS Journal of Photogrammetry and Remote Sensing, vol. 60, No. 2, pp.113-128, 2006.

[3] Tomonori Deguchi, Masatane Kato, Hakan Akcin, Hakan S. Kutoglu, "Monitoring of Mining Induced Land Subsidence using L- and C-band SAR interferometry", pp. 2122-2125, Proceedings IGARSS 2007.

[4] Alex Hay-Man Ng, Linlin Ge, Yueguan Yan, et al. "Mapping accumulated mine subsidence using small stack of SAR differential interferograms in the Southern coalfield of New South Wales", Australia, Engineering Geology, pp1-15, 2010,115

[5] Haertel V. F., Shimabukuro Y. E. "Spectral linear mixing model in low spatial resolution image data", IEEE Transactions on Geoscience and Remote Sensing,pp 2555-2562, 2005,43

[6] Kerdiles H., Grondona M. O.. "NOAA-AVHRR NDVI decomposition and subpixel classification using linear mixing in the Argentinean Pampa", International Journal of Remote Sensing, pp1303-1325, 1995, 16 
[7] Lorenzo Busetto, Michele Meroni, Roberto Colombo. "Combining medium and coarse spatial resolution satellite data to improve the estimation of sub-pixel NDVI time series", Remote Sensing of Environment, pp.118 - 131, 2008, 112

[8] A. R. Huete, H. Q. Liu , K. Batchily, et al. "A comparison of vegetation indices over a global set of TM images for EOSMODIS", Remote Sensing of Environment, pp.440 - 451, 1999, 59

[9] WU Lixin, LI Deren. "Future earth observation cooperation and disaster reduction", Geography and Geo-Information Science, pp. $1-8,2006,22(3)$

[10] HUO Aidi, ZHANG Guangjun, WU Suli, et al. "The application of remote sensing technique to sandy desertification assessment based on MODIS data--Taking North Shaanxi as an example", Agricultural Research in the Arid Areas, pp. 154-158, 2008, 26(2) 